\title{
A Study of Voice Activity Detection Techniques for NIST Speaker Recognition Evaluations
}

\author{
Man-Wai MAK and Hon-Bill YU \\ Centre for Signal Processing, \\ Dept. of Electronic and Information Engineering, \\ The Hong Kong Polytechnic University \\ enmwmak@polyu.edu.hk
}

\begin{abstract}
Since 2008, interview-style speech has become an important part of the NIST Speaker Recognition Evaluations (SREs). Unlike telephone speech, interview speech has lower signal-to-noise ratio, which necessitates robust voice activity detectors (VADs). This paper highlights the characteristics of interview speech files in NIST SREs and discusses the difficulties in performing speech/non-speech segmentation in these files. To overcome these difficulties, this paper proposes using speech enhancement techniques as a preprocessing step for enhancing the reliability of energy-based and statisticalmodel-based VADs. A decision strategy is also proposed to overcome the undesirable effects caused by impulsive signals and sinusoidal background signals. The proposed VAD is compared with the ASR transcripts provided by NIST, VAD in the ETSI-AMR Option 2 coder, satistical-model (SM) based VAD, and Gaussian mixture model (GMM) based VAD. Experimental results based on the NIST 2010 SRE dataset suggest that the proposed VAD outperforms these conventional ones whenever interview-style speech is involved. This study also demonstrates that (1) noise reduction is vital for energy-based VAD under low SNR; (2) the ASR transcripts and ETSI-AMR speech coder do not produce accurate speech and non-speech segmentations; and (3) spectral subtraction makes better use of background spectra than the likelihood-ratio tests in the SM-based VAD. The segmentation files produced by the proposed VAD can be found in http://bioinfo.eie.polyu.edu.hk/ssvad.
\end{abstract}

Keywords: Speaker verification, voice activity detection, NIST SRE, statistical model based VAD, spectral subtraction 


\section{Introduction}

NIST speaker recognition evaluations (SREs) have been focusing on textindependent speaker verification over telephone channels since 1996. In recent years, NIST introduces interview-style speech into the evaluations. For example, the speech files in NIST 2008 SRE contain conversation segments of approximately five minutes of telephone speech and three minutes of interview speech, and the speech files in NIST 2010 SRE contain interview recordings with duration ranging from three to fifteen minutes. In each speech file, about half of the conversation contains speech, and the remaining part contains pauses or silence intervals. The inclusion of non-speech intervals in the speech files necessitates voice activity detection (VAD) because these intervals do not contain any speaker information. In particular, VAD can be used to identify speech segments prior to the feature extraction process.

Speech/Non-speech detection can be formulated as a statistical hypothesis problem aimed at determining to which class a given speech segment belongs. However, a high level of background noise can cause numerous detection errors, because the noise partly or completely masks the speech signal [39]. A robust decision rule that works under noisy conditions is therefore essential. Most of the existing VAD algorithms are effective under clean acoustic environments, but they could fail badly under adverse acoustic conditions [5].

Traditionally, VAD uses periodicity measure [49], zero-crossing rate [4], pitch [10], energy [53], spectrum analysis [36], higher order statistics in the LPC residual domain [37], or combinations of different features [47]. More sophisticated VAD techniques have been proposed for real-time speech transmission on the Internet [43] and mobile communication services [20]. In particular, the adaptive multi-rate (AMR) codec option II (AMR2) [19] uses a decision logic based on the energy of 16 frequency bands, background noise, channel SNR, frame SNR, and long-term SNR [11]. The VAD of this codec takes advantage of speech encoder parameters and is more robust against environmental noise than its earlier version (AMR1) and G.729 [48]. Moreover, the VAD decision threshold can be adapted dynamically according to the acoustic environment, allowing on-line speech/non-speech detection under non-stationary acoustic environments.

More recently, research has focused on statistical-model-based VAD where individual frequency bins of speech are assumed to follow a parametric density function [44]. In this approach, VAD decisions are based on likelihood ratio tests (LRTs) where the geometric mean of the log-likelihood ratios of 
individual frequency bins are estimated from observed speech signals. The statistical model can be Gaussian [44] or generalized Gaussian [24]. However, it has been recently found that Laplacian and Gamma models are more appropriate for handling a wide variety of noise conditions [9]. Using an online version of the Kolmogorov-Smirnov test, the type of models can be selected adaptively for different noise types and SNRs [9]. To improve the robustness of VAD under adverse acoustic environment, contextual information derived from multiple observations has been incorporated into the LRT (MO-LRT) [41]. Gaussian mixture models have been applied to model the static harmonic-structure information and the long-term temporal information of speech. VAD decisions are then based on the log-likelihood ratios computed from the clean and noise GMMs [48, 21]. In [46], Wiener filtering is applied to remove noise before extracting acoustic features for training the speech and non-speech GMMs.

Characteristics of speech and non-speech signals have also been modeled by hidden Markov models (HMMs). For example, in [50], a decision-tree algorithm that combines the scores of HMM-based speech/non-speech models and speech pulse information was used for rejecting far-field speech in speech recognition systems. Both [21, 52] and [50] use statistical models to characterize speech and non-speech signals, with some decision logics governing the switching between speech and non-speech states. The difference being that in the GMM-VAD of [21], state duration is governed by the number of speech frames (as detected by the GMMs) in a fixed-length buffer, and that in the GMM-VAD of [52] state duration is governed by a hangover and handbefore scheme which detects the consonants occurred at the beginning, middle and the end of words; whereas in the HMM-VAD of [50], the state duration is controlled by the state-transition probabilities of the HMMs and speech pulse information. Note that both GMM- and HMM-based VADs require ground-truth speech/non-speech segments for training the statistical models. Unfortunately, these labeled segments are not available in NIST SREs.

The VAD problem has also been formulated as an edge-detection problem. For example, in [30], two optimal 1-D filters with responses invariant to various background noise levels are designed to detect the beginning edges and ending edges of the energy profile of speech signals. To detect the beginning edges, the filter has positive response to a beginning edge, negative response to an ending edge, and near-zero response to silence. The filter for detecting the ending edge has the opposite characteristics and has more time points. The filters are operated as a moving-average filter on the energy envelope and their outputs are compared with dynamic decision thresholds 
estimated from a 2-mode Gaussian mixture model.

In recent NIST SREs, several sites provided the details of their VAD in the system descriptions. Typically, these systems use energy-based methods that estimate a file-dependent decision threshold according to the maximum energy level of the file [28]. Some sites used the periodicity of speech frames or the power of noise-removed speech frames to make speech/non-speech decisions $[26,45,31,54]$. An alternative approach is to use the ASR transcripts supplied by NIST to remove the non-speech segments [12].

In this paper, we propose a VAD that is specifically designed for NIST SREs. Special attention has been paid to address the low SNR, impulsive noise, and cross talks in the interview-style speech files. The main idea is to apply speech enhancement as a pre-processing step to boost the SNR of the speech segments, which facilitates the subsequence speech/non-speech decisions either by log-likelihood ratio tests or comparing with energy-based thresholds. While this strategy has been adopted in the past, e.g., [46, 40, 32], our proposed VAD has some important differences. For example, the VAD in [46] requires the training of speech and non-speech GMMs, whereas ours does not require training. This requirement is a burden for situations like NIST SREs because labeled speech segments are not available. The Wiener filtering in $[46,40]$ and the wavelet denoising in [32] also need to strike a good balance between spectral distortion and the degree of noise removal because decisions of these VADs are based on the spectral features of the noise-reduced speech. Our VAD, on the other hand, does not use the spectral features for VAD decisions. Therefore, it can leverage the oversubtraction to boost the SNR for better discrimination between speech and non-speech. To the best of our knowledge, our study provides the first comprehensive comparison between different VADs for NIST SREs. Results based on NIST 2010 SRE suggest that the proposed VAD outperforms the VAD in AMR2, the transcriptions provided by NIST, and statistical modelbased VAD.

In Section 2, we highlight the characteristics of the interview speech files in NIST SRE and explain why conventional VAD techniques will encounter difficulty in detecting speech in these files. Then, in Section 3, we outline two state-of-the-art statistical VADs and explain how they can be applied to NIST SREs. Section 4 proposes using speech enhancement techniques as a pre-processing step for improving the statistic model based VAD and energy-based VAD. Experimental evaluations comparing different types of VADs under NIST 2010 SRE are then presented in Section 5. 


\section{Characteristics of Interview Speech in NIST SREs}

In early NIST SREs, researchers seldom pay attention to VAD. This is because the telephone speech files in early SREs have high signal-to-noise ratios (SNRs), making VAD a trivial task. The high SNR in telephone speech is resulted from the close proximity between speaker's mouth and the handset. In interview speech, however, different microphone types were used for recording. For example, twelve microphones were used in NIST $2008 \mathrm{SRE}^{1}{ }^{1}$ and in NIST 2010 SRE, the interviewees used different types of far-field microphones, such as lavaliere microphones, camcorders, and hanging microphones [34]. These microphones lead to files with the following characteristics:

1. Low SNR. Depending on the microphone types, some of the interview speech segments have low SNR, causing problems in conventional VAD. Fig. 1(a) shows the waveform of an interview speech file (ftvhv.sph) in NIST 2008 SRE, and Fig. 1(c) highlights a short segment of the same file. The NIST STNR Tool ${ }^{2}$ indicates that the SNR of this file is $5 \mathrm{~dB}$. Although this level of SNR is not very low, it already causes numerous errors in an energy-based VAD, as indicated by "AEVAD" in the lower panel of Fig. 1(c). Fig. 2 shows the histograms of SNR of interview speech files in NIST 2008 and 2010 SRE. While the mean SNRs of these two databases are high $(22 \mathrm{~dB}$ and $21 \mathrm{~dB}$, respectively), about $2 \%$ of the files have SNR less than $5 \mathrm{~dB}$, i.e., about $2 \%$ of the files have situation similar to Fig. 1. The VAD errors in these files will have detrimental effect on speaker verification performance, which will be demonstrated in Section 5 .

2. Impulsive. Some of the files in NIST 2010 SRE contain a large number of spikes that seriously mask the amplitude of speech segments, as illustrated in Fig. 3.

3. Low-energy speech superimposed on periodic background signals. Some files contain low-energy speech superimposed on periodic background noise, as exemplified in Fig. 4.

\footnotetext{
${ }^{1}$ Some of these microphones are of the same models, but they were placed at different positions with respect to the speakers.

${ }^{2}$ This tool is part of the Speech File Manipulation Software(SPHERE) Package Version 2.7, available from http://www.nist.gov/itl/iad/mig/tools.cfm.
} 
4. Cross talk. Each interview speech file in NIST 2010 SRE contains two channels, one recording the speech of an interviewee and the other the speech of an interviewer. As far-field microphones were used for recording interviewee's speech, a low-energy crosstalk signal appears in the interviewee's channel when the interviewer is talking, causing the VAD mistakenly considers the crosstalk as belonging to the interviewee. This situation is exemplified in Fig. 5(a) in which the microphone of the interviewee's channel picks up the speech of the interviewer in Interval A.

As shown in these figures, conventional energy-based VAD fails to detect the speech segments under such conditions.

\section{Statistical Voice Activity Detection}

This section highlights the merit of the statistical model based VAD [44] and GMM-based VAD [21] and explains how they can be applied to detect the speech segments of NIST SRE speech files. The section focuses on the decision logic and threshold determine methods that are specifically designed for the SREs.

\subsection{Statistical Model (SM) Based VAD}

In SM-based VAD [44], speech/non-speech segmentation is formulated as a hypothesis testing problem:

$$
\begin{aligned}
& H_{0}: \text { speech absent }: Y(m)=B(m) \\
& H_{1}: \text { speech present }: Y(m)=X(m)+B(m)
\end{aligned}
$$

where $Y(m), X(m)$, and $B(m)$ represent the DFT of noisy speech, clean speech, and background noise at frame $m$, respectively. The complex DFT coefficients in $Y(m), X(m)$, and $B(m)$ are assumed to be independent and normally distributed. For each frame $m$, a VAD score $\Gamma(m)$ is computed based on the VAD score of the previous frame and the likelihood ratio $\Lambda(m)$ at the current frame:

$$
\Gamma(m)=\frac{P\left(H_{0}\right)}{P\left(H_{1}\right)}\left[\frac{a_{01}+a_{11} \Gamma(m-1)}{a_{00}+a_{10} \Gamma(m-1)}\right] \Lambda(m) \underset{H_{0}}{\stackrel{H_{1}}{\gtrless}} \eta
$$

where $a_{i j} \triangleq \operatorname{Pr}\left(q(m)=H_{j} \mid q(m-1)=H_{i}\right)$ are state-transition probability and $P\left(H_{0}\right)$ and $P\left(H_{1}\right)$ are prior probability. Because DFT coefficients are 
assumed to be independent, we have

$$
\Lambda(m)=\left[\prod_{k=0}^{K-1} \frac{p\left(Y_{k}(m) \mid H_{1}\right)}{p\left(Y_{k}(m) \mid H_{0}\right)}\right]^{\frac{1}{K}}
$$

where $K$ is the number of frequency bins and $p()$ 's are complex normal densities. The VAD score is then compared with a decision threshold $\eta$ to make speech/non-speech decisions.

To apply SM-based VAD to detect speech segments in NIST SRE files, the SM scores $\Gamma(m)$ of the entire utterance are ranked in descending order as shown in Fig. 6. Then, a fixed percentage of scores in the lower and upper ends of the ranked list are selected and assumed to be the background frames and peak frames, respectively. The VAD's fixed decision threshold is a linear combination of the score mean of the lower end $\left(\bar{\Gamma}_{b}\right)$ and the minimum score in the upper end:

$$
\eta=\nu \bar{\Gamma}_{b}+(1-\nu) \min \left\{\Gamma\left(p_{1}\right), \ldots, \Gamma\left(p_{L}\right)\right\},
$$

where $0 \ll \nu<1$ is a weighting factor and $\left\{\Gamma\left(p_{1}\right), \ldots, \Gamma\left(p_{L}\right)\right\}$ are top- $L$ scores. Note that $L$ cannot be too large; otherwise the rank list may include the peaks of some high-energy speech frames, which will lead to underestimation of $\eta$. However, when $L$ is too small, some medium-amplitude spikes will be missed. It was found that the influence of spikes can be largely eliminated by using the minimum amplitude in this ranked list, as evidenced by the VAD result in the Fig. 3 .

The above procedure raises the issue of determining an appropriate percentage for the lower and upper ends of the ranked score list. These percentages can be founded by inspecting several interview speech files in NIST 2005-2008 SREs. By examining some of these files, we found that it is fairly safe to assume that among all the frames in a speech file, $10 \%$ are background frames and $5 \%$ contain signal peaks.

\subsection{Gaussian Mixture Model (GMM) Based VAD}

Mel-frequency cepstral coefficients (MFCCs) are known to be inadequate for discriminating speech and non-speech frames, primarily because of the similarity between the static MFCC vectors of speech and background noise. On the other hand, the harmonic structures of speech and background noise are more distinguishable and more noise robust [25]. Based on this argument, Fukauda et al. [21] extracted the harmonic-structure-based features 
from the middle range of the cepstral coefficients obtained from the discrete cosine transform (DCT) of the power spectral coefficients.

The cepstral coefficients $c_{i}(m)$ with small and large indexes $i$ are liftered out because they include long and short oscillations. On the other hand, the coefficients in the middle part of the cepstrum capture the harmonic structure information in the human voice. The liftered cepstrum $\hat{c}_{i}(m)$ is converted back to the log power spectrum by inverse DCT, followed by the exponential transform to obtain the linear power spectrum. The power spectrum are finally converted to mel-cepstrum $\hat{q}_{n}(m)$ by applying a melscale filter bank and DCT, where $n$ is the bin number of the harmonic structure-based mel cepstral coefficients. This feature captures the envelope information of the local peaks in the frequency spectrum corresponding to the harmonic information in the speech signals. Fig. 7 shows the procedure of extracting the harmonic-structure-based features.

Dynamic (spectro-temporal) features capture the variation of the spectral envelopes along the time axis. They are typically obtained by estimating the derivative of 5 to 9 consecutive acoustic vectors. The first-order derivative of a sequence of cepstral vectors is called delta cepstrum, and the second-order derivative is called delta-delta cepstrum.

In GMM-based VAD, the speech/non-speech decision at frame $m$ is given by the log-likelihood ratio

$$
\mathcal{L}(m)=\log p\left(\boldsymbol{y}(m) \mid H_{1}\right)-\log p\left(\boldsymbol{y}(m) \mid H_{0}\right) \underset{H_{0}}{\stackrel{H_{1}}{\gtrless}} \eta,
$$

where the acoustic vectors $\boldsymbol{y}$ 's are assumed to follow a mixture of Gaussian distribution:

$$
p\left(\boldsymbol{y} \mid H_{i}\right)=\sum_{j=1}^{J} w_{i j} \mathcal{N}\left(\boldsymbol{y} ; \boldsymbol{\mu}_{i j}, \boldsymbol{\Sigma}_{i j}\right)
$$

where $w_{i j}, \boldsymbol{\mu}_{i j}$ and $\boldsymbol{\Sigma}_{i j}$ are the mixture weights, mean vectors and covariance matrices for either speech $(i=1)$ or non-speech $(i=0)$ model.

The decision threshold $\eta$ is determined by a strategy similar to that of SM-VAD (Eq. 4) described in Section 3.1. Specifically, 20\% and 5\% of a speech file are assumed to contain background frames and signal peaks, respectively.

Unlike the SM-based VAD, the GMM-based VAD requires the training of two GMMs - one representing speech and another one representing nonspeech. This means that some speech files with speech and non-speech segmentations are required. In theory, the segmentations had better be 
the ground-truths, i.e., they need to be done by listening tests and human inspections of spectrograms. This is not a problem if clean speech files are available and the VAD is tested on the same files with noise added to them, e.g., the experiments in [21]. However, in NIST SREs, the requirement of ground-truth segmentations will cause difficulty because no clean speech files are available for the listening tests. Even if we can find some interview-style speech files with high enough SNR for the listening tests, they may be too clean and therefore cannot represent the realistic situations in other noisy speech files. Furthermore, hand labeling a large number of speech files is too laborious and time-consuming.

Here, we propose an automatic method that can find speech and nonspeech segments that are close enough to the ground-truths for training the GMMs. Fig. 8 shows the procedure. Unlike the VAD in [21], our GMMbased VAD contains an extra processing block - Frame Index Extraction that finds the frame indexes of speech and non-speech segments with very high confidence of being correct. This seems to be a chicken-and-egg problem because if a reliable VAD exists, we do not need to build a new one in the first place. However, having some reliable speech and non-speech segments does not mean that we need a reliable VAD to detect both at the same time. The idea is that we can always make a simple energy-based VAD very reliable in detecting speech but extremely unreliable in detecting non-speech by adjusting the decision threshold such that it can achieve a very low false alarm (consider non-speech as speech) but having a very high missing rate (consider speech as non-speech). A similar argument applies to the reliable detection of non-speech. Because this simple VAD can only maintain either the false alarm or missing rate low but not both, it can only be used as a pre-processing step in more sophisticated VADs such as the one illustrated in Fig. 8.

The idea is to leverage the large number of speech files in NIST SREs. Specifically, for each interview-style speech files in the training set (e.g., past NIST SREs), a simple energy-based VAD is used to determine the energy of all frames. Then, the frames are ranked in ascending order of energy as illustrated in Fig. 9. The top 5\% of the ranked list are discarded because the high energy is most likely caused by spiky signals instead of speech. Because of the simplicity of the energy-based VAD, there will be many false alarms and misses in the detections. Therefore, only a small percentage in the upper- and lower-part of the ranked list are considered as speech and non-speech, respectively. More precisely, $99 \%$ of the frames in the middle of the ranked list will be discarded, and only the frames with a very high confidence of having a correct segmentation are retained for training the 
GMMs.

Given the frame indexes of speech and non-speech segments, static harmonic features and long-term dynamic features are extracted and concatenated, forming two streams of feature vectors as shown in Fig. 8. These concatenated features vectors are then used to train the GMMs. In this work, 3,569 interview-style utterances from NIST SRE 2005-2008 were used for training the GMMs. This amount to 280,010 training vectors per GMM. The number of mixtures $J$ was set to 32 , and all Gaussians have a full covariance matrix.

One advantage of the GMM-based VAD is that it is less susceptible to spiky signals because these signals have low-level of harmonic contents and their temporal property is also different from that of speech signals. However, GMM-based VAD also has its own limitations. In particular, because the GMM-based VAD does not rely on SNR, it may falsely detect some weak cross-talks from other speakers as speech segments as long as the cross-talks contain speech-like characteristics. This drawback can be alleviated by using spectral subtraction as a pre-processor because the weak cross-talks will be considered as background signals so that they can be largely eliminated in the spectral subtraction process. Further discussions on the use of spectral subtraction as a pre-processor can be found in the next section.

\section{Speech Enhancement for VAD}

Noise removal is a vital step for pre-processing the interview speech files in NIST SREs because many of them have low SNR. This paper proposes to apply spectral subtraction (SS) with a large over-subtraction factor to discard the background noise as much as possible before passing the enhanced speech to an energy-based VAD. Advanced speech enhancement techniques (e.g. MMSE [16] and LSA-MMSE [17]) have not been used because audio quality of reconstructed speech is not the main concern. ${ }^{3}$ Instead, it is more important to increase the SNR in speech regions and to minimize the background noise in non-speech regions. Spectral subtraction meets this requirement without unnecessarily complicating the whole system.

\footnotetext{
${ }^{3}$ Acoustic features (MFCCs) were extracted from the original signals instead of from the reconstructed signals.
} 


\subsection{Noise Reduction via Spectral Subtraction}

To obtain the enhanced speech $\hat{x}(t)$ from the noisy speech $y(t)$ at frame $m$, we implemented the spectral subtraction $[6,15,51]$ of the form

$$
\hat{X}_{k}(m)= \begin{cases}{\left[\left|Y_{k}(m)\right|-\alpha(m)\left|\hat{B}_{k}\right|\right] e^{j \varphi_{k}(m)}} & \text { if }\left|Y_{k}(m)\right|>(\alpha(m)+\beta(m))\left|\hat{B}_{k}\right| \\ \beta(m)\left|\hat{B}_{k}\right| e^{j \varphi_{k}(m)} & \text { otherwise, }\end{cases}
$$

where $k$ is the frequency bin index, $\varphi_{k}(m)$ is the phase of $Y_{k}(m), \hat{B}_{k}$ is the average spectrum of some non-speech regions, $\alpha(m)$ is an over-subtraction factor for removing background noise, and $0<\beta(m) \ll 1$ is a spectral floor factor ensuring that the recovered spectra never fall below a preset minimum. The value of $\alpha(m)$ and $\beta(m)$ are computed as

$$
\begin{aligned}
& \alpha(m)=-\frac{1}{2} \gamma(m)+c \quad\left(\alpha_{\min } \leq \alpha(m) \leq \alpha_{\max }\right) \\
& \beta(m)= \begin{cases}\beta_{\min } & \text { if } \gamma(m)<1 \\
\beta_{\max } & \text { otherwise }\end{cases}
\end{aligned}
$$

where $\gamma(m)=\frac{\sum_{k}\left|Y_{k}(m)\right|}{\sum_{k}\left|\hat{B}_{k}\right|}$ is the a posteriori SNR, $c$ is a constant $(=4.5$ in this work), $\alpha_{\min }, \alpha_{\max }, \beta_{\min }$, and $\beta_{\max }$ constrain the allowable range of the over-subtraction factor and the noise floor. We set these values such that the speech spectra are over-subtracted when the SNR is low. In this work, we set $\alpha_{\max }=4, \alpha_{\min }=0.5, \beta_{\max }=0.05$, and $\beta_{\min }=0.01$. These values were determined empirically through experimentations on an i-vector systems (see Section 5.5) and by visual comparison between the original and reconstructed waveforms of several speech files. Because of the small $\beta_{\max }(\ll 1)$, musical noise occurs when some frequency components meet the condition in the upper branch of Eq. 7 while some others do not. While musical noise appears in both speech and non-speech regions, its energy in non-speech regions is not high enough to cause false detections, as evident in Fig. 1(d). Also, although this musical noise will degrade the perceptual quality of the denoised speech, it is not a concern here because the denoised speech is only used for $\mathrm{VAD}$, not for speaker recognition, i.e., our goal is to detect voice activity rather than speech enhancement.

Note that if the background noise is high, consonants with weak energy will be masked by the noise. Therefore, it is more appropriate to exclude these weak consonants by means of over subtraction. On the other hand, if the background noise is low, $\left|\hat{B}_{k}\right|$ in Eq. 7 is almost zero, meaning that consonants will also be included. 
Fig. 2 shows the histograms of the speech files in 2008 and 2010 SREs before and after spectral subtraction. Evidently, spectral subtraction can improve the SNR significantly.

\subsection{Threshold Determination and VAD Decision Logic}

Fig. 10 shows the structure of the proposed energy-based VAD, which we refer to as SS+AE-VAD. For each utterance, after noise removal, the energy of each 10-ms frame is computed at every $1 \mathrm{~ms}$. To avoid excessive fluctuation in the energy profile, a 40-tap moving average filter is applied to smooth the profile.

The presence of spikes in some files affects the maximum SNR in these files, which needs to be taken care of when determining the VAD decision threshold. In particular, these spikes lead to overestimation of the decision threshold if it is based on the background amplitude and the maximum amplitude. Consequently, low-energy speech segments could be mistakenly detected as non-speech. To address this problem, we have developed a similar strategy as the one in Section 3.1, but considering signal amplitude rather than statistical scores. The decision threshold is a linear combination of the mean of background amplitude $\left(\bar{a}_{b}\right)$ and the minimum of the signal peaks:

$$
\eta=\nu \bar{a}_{b}+(1-\nu) \min \left\{a\left(p_{1}\right), \ldots, a\left(p_{L}\right)\right\},
$$

where $\left\{a\left(p_{1}\right), \ldots, a\left(p_{L}\right)\right\}$ are the amplitudes (after the moving average filter) of $L$ largest-amplitude frames. In this work, $L$ was set to $1 \%$ of the total number of frames in the speech file. By comparing the amplitude of each frame in the file with the threshold, those frames with amplitude larger than the threshold are considered as speech frames.

Figs. 1(b) and (d) show the same speech file and segment as in Figs. 1(a) and (c) but after spectral subtraction. Evidently, with the background noise largely removed, speech and non-speech intervals can be correctly detected by an energy-based VAD. To highlight the advantage of spectral subtraction, Figs. 1(c) and 1(d) compare the segmentation results of SS+AE-VAD and that of the ETSI-AMR coder (Option 2). The figure suggests that this coder over-estimates the length of speech segments, whereas the $\mathrm{SS}+\mathrm{AE}-$ VAD correctly detects the speech segments.

\section{Experiments and Results}

VAD algorithms are typically evaluated by comparing the VAD decisions on clean speech against the VAD decisions on noise contaminated speech 


\begin{tabular}{|c|c|c|}
\hline \multicolumn{2}{|r|}{ VAD } & Description \\
\hline 1 & AE-VAD & $\begin{array}{l}\text { Energy-based VAD with the decision governed by the combination be- } \\
\text { tween average magnitude of background noise and signal peaks. The } \\
\text { combination is controlled by a weighting factor }(\nu \text { in Eq. } 9) \text {. }\end{array}$ \\
\hline 2 & ASR-VAD & $\begin{array}{l}\text { Speech segments in the Automatic Speech Recognition transcripts pro- } \\
\text { vided by NIST [34]. }\end{array}$ \\
\hline 3 & AMR-VAD & VAD in ETSI Adaptive Multi-Rate coder (Option2) [18]. \\
\hline 4 & SM-VAD & $\begin{array}{l}\text { Sohn's statistical-model-based VAD [44] incorporated with a fixed } \\
\text { threshold, determined by Eq. } 4 \text {. }\end{array}$ \\
\hline 5 & GMM-VAD & $\begin{array}{l}\text { Gaussian-mixture-model-based VAD using long-term temporal informa- } \\
\text { tion and harmonic structure-based features in noisy speech [21] incor- } \\
\text { porated with a fixed decision threshold. }\end{array}$ \\
\hline 6 & SS+SM-VAD & SM-VAD with spectral subtraction as a pre-processing step. \\
\hline 7 & SS+AE-VAD & AE-VAD with spectral subtraction as a pre-processing step. \\
\hline
\end{tabular}

Table 1: The voice activity detection (VAD) methods being applied in this paper and their acronym.

[3], with performance shown on a receiver operating characteristic (ROC) curve. However, the noisy speech files in NIST SREs do not have their clean counterparts. Instead of hand labeling thousands of speech files, we used the performance indexes of speaker verification, i.e. equal error rate (EER), detection error tradeoff (DET) curves, and minimum normalized decision cost function (DCF) for quantifying VAD performance. Discussions and explanations of these performance indexes can be found in [33] and the evaluation plans of NIST SRE. ${ }^{4}$

The experiments involve seven VADs, as shown in Table 1. Among the five conventional VADs (VADs 1-5), we applied spectral subtraction to the best performing (SM-VAD) and the worst performing (AE-VAD) ones, resulting in SS+SM-VAD and SS+AE-VAD in the last two rows of Table 1. By comparing the speaker verification performance obtained by these VADs against the ones without spectral subtraction, we can observe the contribution of spectral subtraction to the VAD performance.

\subsection{Speech Data}

NIST 2005-2010 SREs were used in the experiments. NIST'05-08 SREs were used as development data, and NIST'10 was used for performance eval-

\footnotetext{
${ }^{4}$ http://www.itl.nist.gov/iad/mig/tests/spk/2010/index.html
} 
uations. Only male speakers in these corpora were used. The core task of NIST'10 is divided into 9 common conditions. Conditions 1, 2, 4, 7 and 9 were considered because interview speech and telephone speech collected by different microphones are involved in these five conditions. Detail descriptions of these five conditions can be found in Section 4 of [35].

\subsection{Speaker Modeling and Channel Mismatch Compensation}

The target-speakers were modeled by GMM-SVM [7] and i-vectors [14].

For the GMM-SVM systems, we extracted 12 MFCCs [13] and their first derivatives from the speech regions of the utterances to create 24dim acoustic vectors. Cepstral mean normalization [1] was applied to the MFCCs, followed by feature warping [38]. A 512-center gender-dependent universal background model was created by using the interview utterances of NIST'05-06. MAP adaptation [42], with relevance factor set to 16, was then performed for each of the target-speakers to create target-dependent GMMs. The same MAP adaptation was also applied to 300 background speakers (also from NIST'05-06) to create 300 impostor GMMs. The mean vectors of these GMMs were stacked to produce 12288-dim GMM-supervectors [7]. Finally, a GMM-SVM speaker model for each target speaker is trained by using his target-dependent GMM-supervector and the background GMMsupervectors. The utterances of 144 male speakers from NIST'05-08 were used for estimating the gender-dependent nuisance attribute projection (NAP) matrices [8] to reduce channel effects (NAP corank was set to 128). Each of these 144 speakers has at least 8 utterances. For the T-norm speaker models [2], 300 male utterances from NIST'05 were used. ${ }^{5}$ The same set of background speakers used for creating the target-speaker SVMs were used for creating the T-norm SVMs.

For the i-vector systems, 19 MFCCs together with energy plus their 1stand 2nd- derivatives were extracted from the speech regions as detected by the VADs, followed by cepstral mean normalization and feature warping with a window size of 3 seconds. A 60-dim acoustic vector was extracted every $10 \mathrm{~ms}$, using a Hamming window of 25ms. NIST 2006-2008 microphone data were used to train a 1024-center UBM. We selected 6,102 utterances from 191 speakers in NIST 2005-2008 SREs to estimate a total variability matrix with 400 total factors. Then, we used 9511 utterances of these 191 speakers

\footnotetext{
${ }^{5}$ It was drawn to the authors' attention that performance could be improved if impostors and T-norm speakers from NIST'08 were used because they match the test speakers in NIST'10 better.
} 


\begin{tabular}{|c|c|c|c|c|c|c|c|c|c|c|c|c|}
\hline \multirow[b]{2}{*}{ VAD Method } & \multicolumn{6}{|c|}{ Equal Error Rate (\%) } & \multicolumn{6}{|c|}{ Minimum Normalized DCF } \\
\hline & CC1 & $\mathrm{CC} 2$ & $\mathrm{CC} 4$ & CC7 & CC9 & Overall & CC1 & $\mathrm{CC} 2$ & CC4 & CC7 & CC9 & Overall \\
\hline AE-VAD & 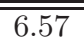 & $\overline{11.72}$ & $\overline{7.23}$ & $\overline{12.28}$ & 7.44 & 10.30 & 0.84 & 0.99 & 0.96 & 0.84 & 0.97 & 0.97 \\
\hline ASR-VAD & 5.15 & 8.58 & 7.74 & 12.81 & 5.74 & 8.88 & 0.78 & 0.85 & 0.74 & 0.88 & 0.77 & 0.90 \\
\hline AMR-VAD & 4.44 & 8.05 & 9.44 & 12.85 & 5.98 & 9.61 & 0.81 & 0.85 & 0.80 & 0.77 & 0.55 & 0.90 \\
\hline GMM-VAD & 3.64 & 5.68 & 5.71 & 8.93 & 4.27 & 6.28 & 0.71 & 0.72 & 0.72 & 0.63 & 0.45 & 0.82 \\
\hline SM-VAD & 3.23 & 4.68 & 4.49 & 9.48 & 3.06 & 5.03 & 0.66 & 0.68 & 0.70 & 0.65 & 0.38 & 0.77 \\
\hline SS+SM-VAD & 2.83 & 4.45 & 4.04 & 7.58 & 2.56 & 4.80 & 0.62 & 0.61 & 0.70 & 0.59 & 0.42 & 0.76 \\
\hline SS+AE-VAD & 2.82 & 4.44 & 3.51 & 6.70 & 2.37 & 4.55 & 0.70 & 0.58 & 0.62 & 0.64 & 0.17 & 0.72 \\
\hline
\end{tabular}

Table 2: Performance of GMM-SVM systems based on 7 VADs under Common Conditions (CC) 1, 2, 4, 7 and 9 of NIST 2010 SRE (male speakers). Refer to Table 1 for the definition of the VADs.

to estimate the Gaussian PLDA [22] loading matrix with 150 latent variables. We applied length normalization [22] to all i-vectors before computing the loading matrix.

\subsection{Selection of Threshold Parameters for $S S+A E-V A D$}

As mentioned in Section 4, energy-based VAD requires a decision threshold for making speech/non-speech decisions. An experiment was conducted to investigate the effect of varying the weighting factor $\nu$ (Eq. 9) on the energy-based VAD.

Fig. 11 suggests that the best range of $\nu$ in Eq. 9 is between 0.95 and 0.99 . Once this value drops below 0.95 , the performance degrades rapidly. This implies that the peak amplitudes can only be used as a reference for setting the VAD decision threshold, whereas the background amplitudes are more trustworthy. However, the threshold cannot totally relies on the background amplitude, because the EER and minDCF increase when $\nu$ increases from 0.99 to 1.0 .

\subsection{Comparing Different VADs}

Based on the results in Section 5.3, the weighting factor $\nu$ in Eq. 9 was set to 0.95 and 0.96 for AE-VAD and SS+AE-VAD, respectively. For SM-VAD, $\mathrm{SS}+\mathrm{SM}-\mathrm{VAD}$, and GMM-VAD, $\nu$ in Eq. 4 was set to 0.993 .

Table 2 shows the equal error rate (EER) and minimum normalized decision cost function (minNDCF) achieved by the GMM-SVM systems. The 
results suggest that preprocessing the noisy sound files by spectral subtraction is a promising idea. After applying SS, the AE-VAD and SM-VAD reduce the overall EER by $56 \%$ and $5 \%$ respectively.

Fig. 12 shows the DET performance achieved by the seven VADs. The results show that SS+AE-VAD achieves a significant lower error rates than the ETSI-AMR coder, ASR transcripts and the simple energy-based VAD for a wide range of operating points.

The results shows that SM-VAD performs better than GMM-VAD for detecting interview speech in NIST SREs. Note that this result does not mean that SM-VAD is better than GMM-VAD for all tasks. In fact, the conditions in NIST SREs are disadvantageous to GMM-VAD because the GMMs of this VAD requires a large number of ground-truth speech and nonspeech segments for training. Unfortunately, in NIST SREs, such segments are not available. Therefore, we developed an automatic approach (see Section 3.2) to finding a large number of speech and non-speech segments that are close enough to the ground-truth as substitutions. The SM-VAD, on the other hand, does not require any ground-truth segments. Therefore, it is more appropriate for NIST SREs.

We notice that both SS and SM work well for the interview speech in NIST 2010 SRE. The error rates achieved by SS+AE-VAD, however, are slightly lower than that achieved by SM-VAD.

Comparing the results of AE-VAD and SS+AE-VAD reveals that SS has significant contribution to the conventional energy-based VAD. However, the performance of SS+SM-VAD is only slightly better than that of SM-VAD. This suggests that SS is not vital to the statistical-model-based VAD. The reason is that in SM-based VADs, the background spectrum has already been taken into account in the scoring function. As pre-processing the noisy speech by spectral subtraction is another approach to using the background spectrum, therefore in SS+SM-VAD, the background spectrum has been used twice. As a result, the gain of applying SS to SM-VAD is not as significant as applying SS to AE-VAD.

Note that SS+AE-VAD and SM-VAD use the background spectrum in a different manner. For the former, the background spectrum is used for spectral subtraction, whereas for the latter it is used for computing the likelihood ratio scores. This difference enables us to make better use of the background spectrum in SS+AE-VAD. Specifically, to remove as much background noise as possible, we may apply a large upper-limit for the oversubtraction factor $\left(\alpha_{\max }\right)$ and a small lower-limit for the noise floor $\left(\beta_{\min }\right)$. The over-subtraction factor $\alpha(m)$ is a linear function of the a posteriori SNR for certain range of SNR and is bounded by the lower- and upper-limit when 
the SNR is beyond this range. As a result, more background noise will be removed in low SNR region whereas more speech content will be retained in high SNR region. The SM-VAD, on the other hand, does not have such property because the background spectrum is assumed constant for both low and high SNR.

The results show that using the ASR transcripts provided by NIST SRE Workshop as VAD leads to poor speaker verification performance, suggesting that the ASR transcripts do not produce accurate speech/non-speech segmentations. The VAD in ETSI-AMR coder also performs poorly. This is mainly caused by the overestimation of both the speech onset and offset regions. To ensure the intelligibility of the encoded speech, it is important for the VAD in a speech coder to include speech onsets and offsets. However, this overestimation is not appropriate for speaker verification, as excessive amount of non-speech will be used for verification.

\subsection{Performance of I-Vector Systems}

While Table 2 suggests that spectral subtraction is an appropriate preprocessing step for both energy-based VAD and statistical-model based $\mathrm{VAD}$, it is of interest to investigate if spectral subtraction is also suitable for state-of-the-art i-vector systems. To further compare the performance of the proposed VAD with more advanced statistical VADs, the distribution of DFT coefficients in SM-VAD were assumed to follow not only Gaussian distributions but also Laplacian and Gamma distributions, similar to that of $[9]$.

The performance of SM-VAD (Gaussian) with and without spectral subtraction is shown in Table 3. Evidently, spectral subtraction can help the SM-VAD. Results also show that SM-VAD based on Gamma distributions performs slightly better than Sohn's classical SM-VAD in terms of EER, but in terms of minimum DCF, Sohn's SM-VAD peforms better.

Recall from Eq. 8 that there are several parameters in spectral subtraction. It is of interest to see if the performance of SS+AE-VAD is sensitive to these parameters. Among the parameters in this VAD, $\alpha_{\max }$ and $\beta_{\max }$ have the greatest impact on the denoised waveform. Therefore, we varied these two parameters and investigated how they affect the performance of the i-vector systems. As shown in Table 3, the performance of SS+AE-VAD is the best when $\alpha_{\max }=4$ or 8 and $\beta_{\max }=0.05$, which also agree with the configuration we used for the GMM-SVM system in Table 2.

As $\alpha_{\max }$ determines the maximum amount of noise to be subtracted from the noisy signal, the good performance at large value of $\alpha_{\max }$ in Table 3 


\begin{tabular}{|c|c|c|c|c|c|c|c|}
\hline \multirow{2}{*}{\multicolumn{2}{|c|}{ Method }} & \multicolumn{3}{|c|}{ Equal Error Rate (\%) } & \multicolumn{3}{|c|}{ Minimum Normalized DCF } \\
\hline & & CC1 & $\mathrm{CC} 2$ & CC1 \& 2 & CC1 & $\mathrm{CC} 2$ & CC1 \& 2 \\
\hline \multicolumn{2}{|c|}{ SM-VAD (Gaussian) } & 1.82 & 3.14 & 3.02 & 0.295 & 0.468 & 0.487 \\
\hline \multicolumn{2}{|c|}{ SM-VAD (Laplacian) } & 2.02 & 2.90 & 2.92 & 0.299 & 0.485 & 0.536 \\
\hline \multicolumn{2}{|c|}{ SM-VAD (Gamma) } & 1.92 & 2.91 & 2.87 & 0.314 & 0.487 & 0.540 \\
\hline \multicolumn{2}{|c|}{ SS+SM-VAD (Gaussian) } & 1.48 & 2.31 & 2.30 & 0.315 & 0.483 & 0.511 \\
\hline \multirow{4}{*}{$\begin{array}{l}\mathrm{SS}+\mathrm{AE}-\mathrm{VAD} \\
\left(\beta_{\max }=0.05\right)\end{array}$} & $\alpha_{\max }=1$ & 2.93 & 4.76 & 4.43 & 0.395 & 0.665 & 0.617 \\
\hline & $\alpha_{\max }=2$ & 1.40 & 2.59 & 2.54 & 0.353 & 0.466 & 0.512 \\
\hline & $\alpha_{\max }=4$ & 1.60 & 2.68 & 2.55 & 0.351 & 0.463 & 0.470 \\
\hline & $\alpha_{\max }=8$ & 1.60 & 2.68 & 2.55 & 0.351 & 0.463 & 0.470 \\
\hline \multirow{2}{*}{$\begin{array}{c}\mathrm{SS}+\mathrm{AE}-\mathrm{VAD} \\
\left(\alpha_{\max }=4\right)\end{array}$} & $\beta_{\max }=0.1$ & 1.69 & 2.68 & 2.55 & 0.356 & 0.458 & 0.481 \\
\hline & $\beta_{\max }=0.5$ & 4.35 & 4.93 & 4.92 & 0.354 & 0.493 & 0.518 \\
\hline
\end{tabular}

Table 3: Performance of an i-vector system based on statistical-model based VAD (SM-VAD) with three different distributions of DFT coefficients and spectral-subtraction VAD (SS+AE-VAD and SS+SM-VAD) under the interview-interview conditions of NIST 2010 SRE.

confirms our earlier argument in Section 4.1 that it is desirable to use oversubtraction to remove as much noise as possible when the SNR is low.

\section{Conclusions and Future Work}

A voice activity detector specially designed for extracting speech segments from the interview-speech files in NIST SREs has been proposed and evaluated under the NIST 2010 SREs protocols. Several conclusions can be drawn from this work: (1) noise reduction is of primary importance for energy-based VAD under low SNR; (2) it is important to remove the sinusoidal background noise as this kind of background signal could lead to many false detection in energy-based VAD; (3) a reliable threshold strategy is required to address the spiky (impulsive) speech signals; and (4) our proposed spectral subtraction VAD outperforms the segmentations derived from the ASR transcripts provided by NIST, the VAD in the advanced speech coder (ETSI-AMR, Option2), the state-of-the-art statistical-model-based VAD, and Gaussian-mixture-model-based VAD in speaker verification.

The proposed VAD is optimized for interview speech in NIST SREs. It is of interest to investigate its performance on other databases, including those in speech recognition such as CENSREC-1-C speech database [29] and Aurora 2 database [27]. The present study assumes that background noise is stationary. It is of interest to apply methods - such as [23] - that can deal with non-stationary noises in NIST SREs. 


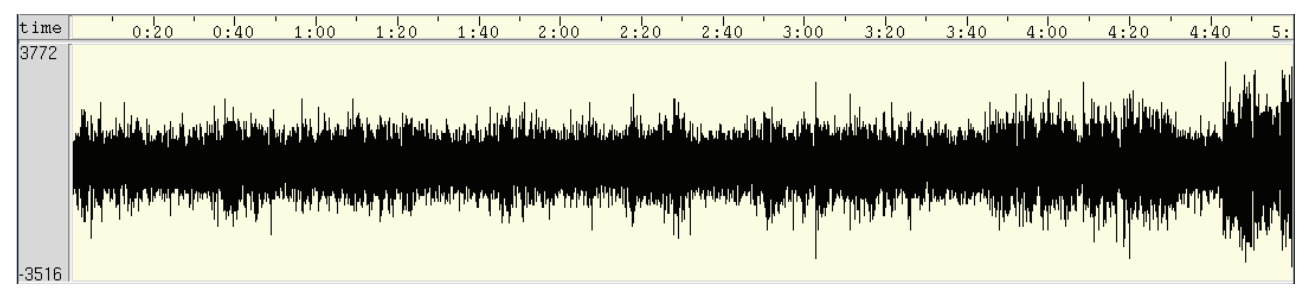

(a) The whole speech file (without denoising)

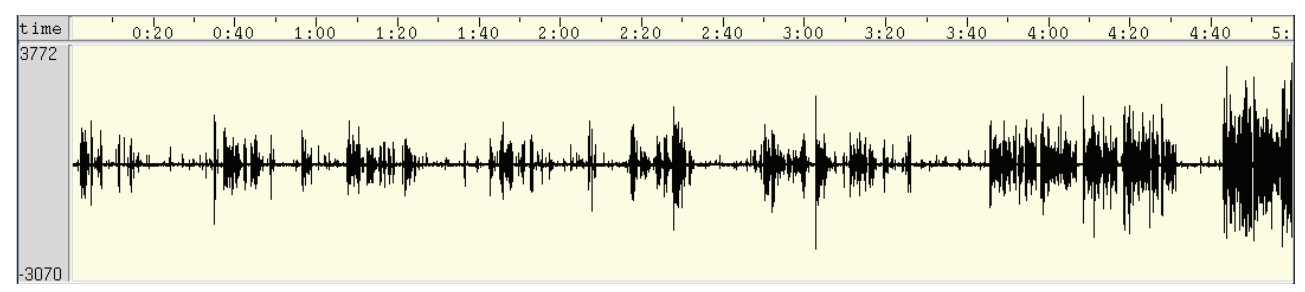

(b) The whole speech file (with denoising)

Figure 1: Waveform, spectrogram, and speech/non-speech decision of an energybased VAD and the ETSI-AMR coder on an interview speech file. [(a) and (c)] without denoising. [(b) and (d)] with denoising. The VAD decisions (S for speech and $\mathrm{N}$ for non-speech) are shown in the bottom panels of (c) and (d). See Table 1 for the abbreviations of the VADs. 


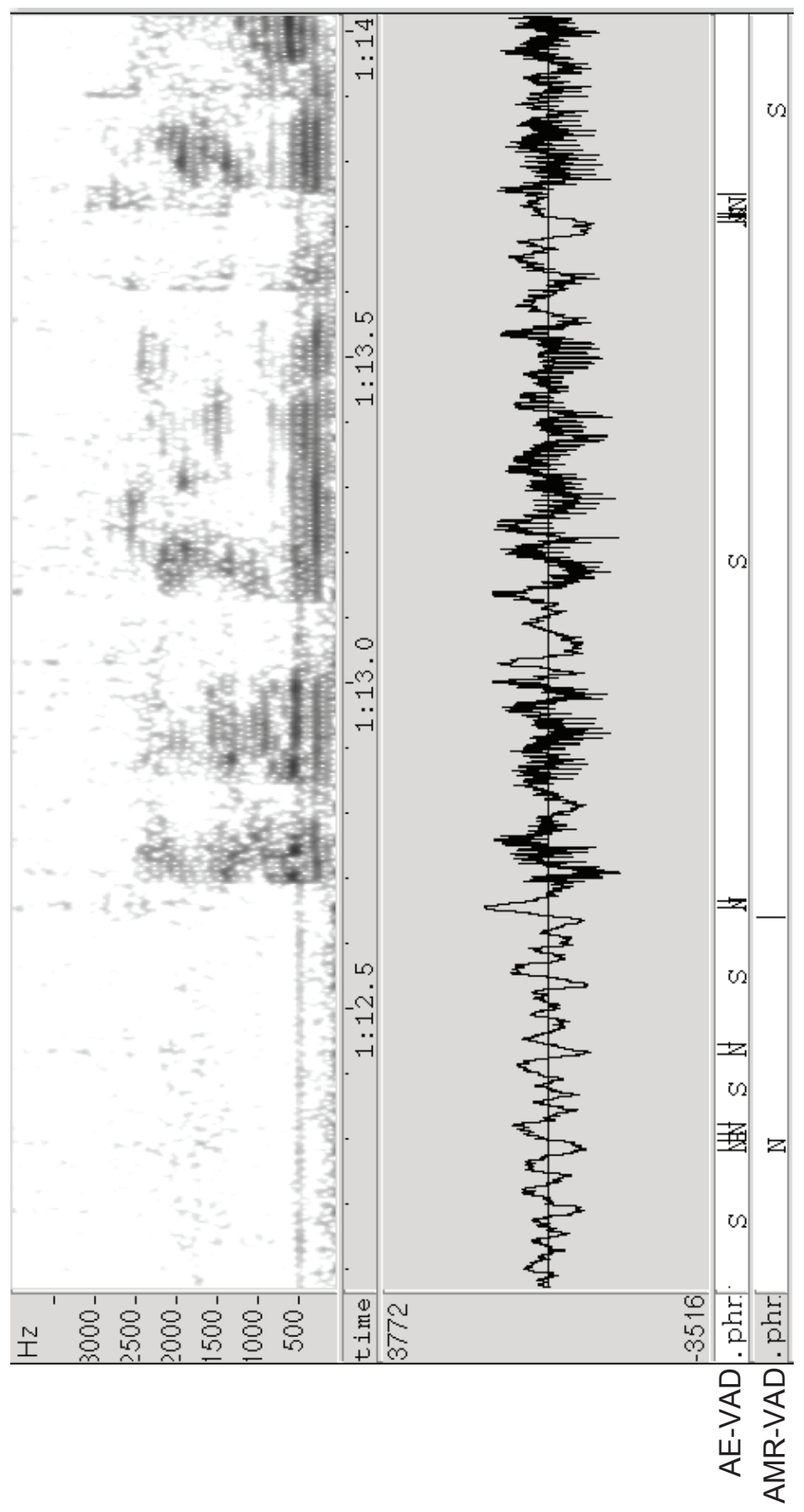

(c) A short segment (without denoising)

Figure 1: (continued) 


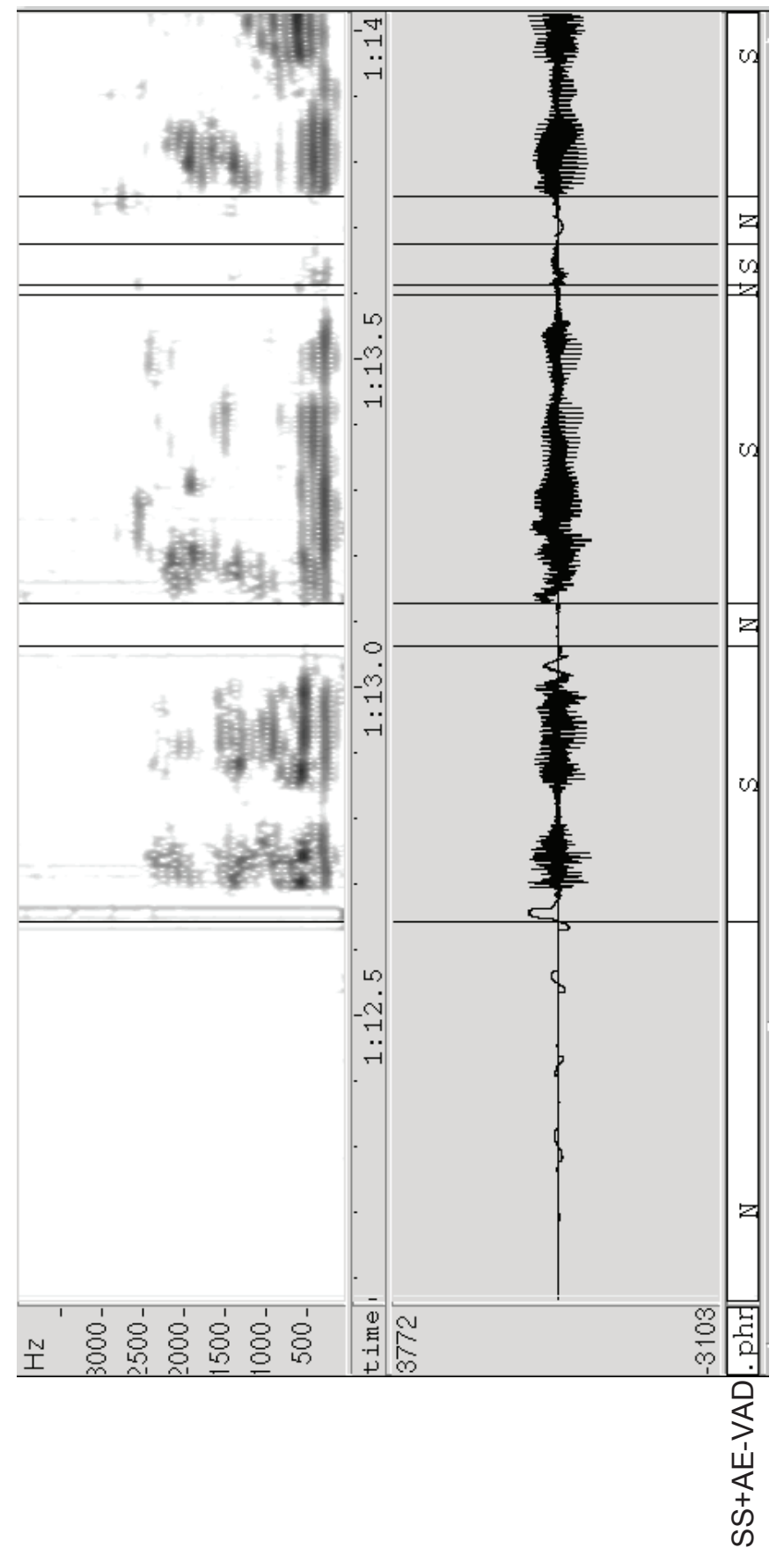

(d) A short segment (with denoising)

Figure 1: (continued) 


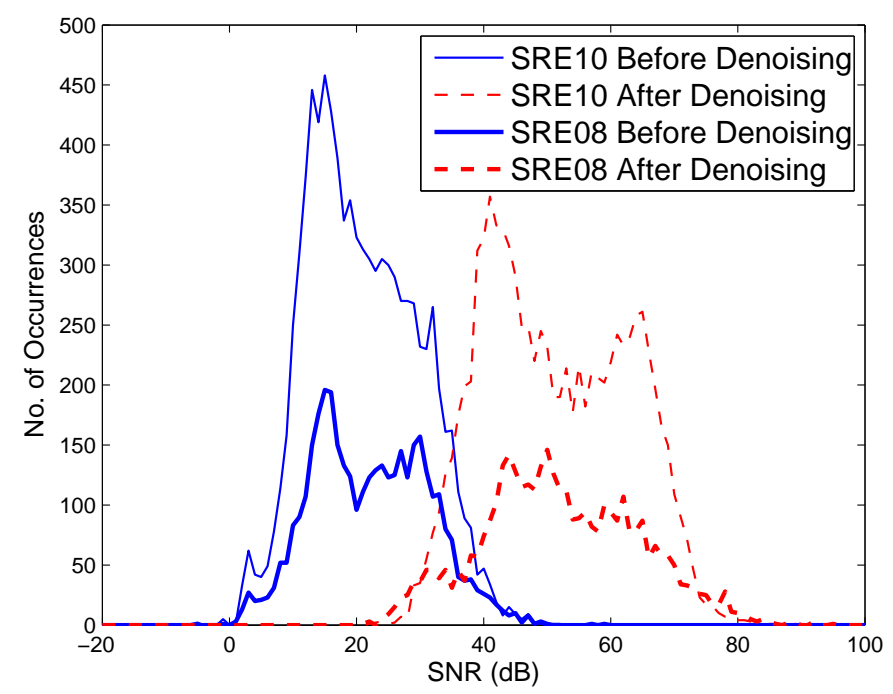

Figure 2: Histograms of SNR of interview speech files in NIST 2008 and 2010 SREs before and after spectral subtraction. The SNRs were measured by the NIST STNR Tool.

\section{Acknowledgement}

This work was in part supported by The Hong Kong Research Grant Council, Grant No. PolyU5251/08E and PolyU5264/09E.

\section{References}

[1] Atal, B.S., 1974. Effectiveness of linear prediction characteristics of the speech wave for automatic speaker identification and verification. J. Acoust. Soc. Am. 55, 1304-1312.

[2] Auckenthaler, R., Carey, M., Lloyd-Thomas, H., 2000. Score normalization for text-independent speaker verification systems. Digital Signal Processing 10, 42-54.

[3] Basbug, F., Nandkumar, S., Swaminathan, K., 1999. Robust voice activity detection for DTX operation of speech coders, in: IEEE Workshop on Speech Coding, pp. 58-60. 


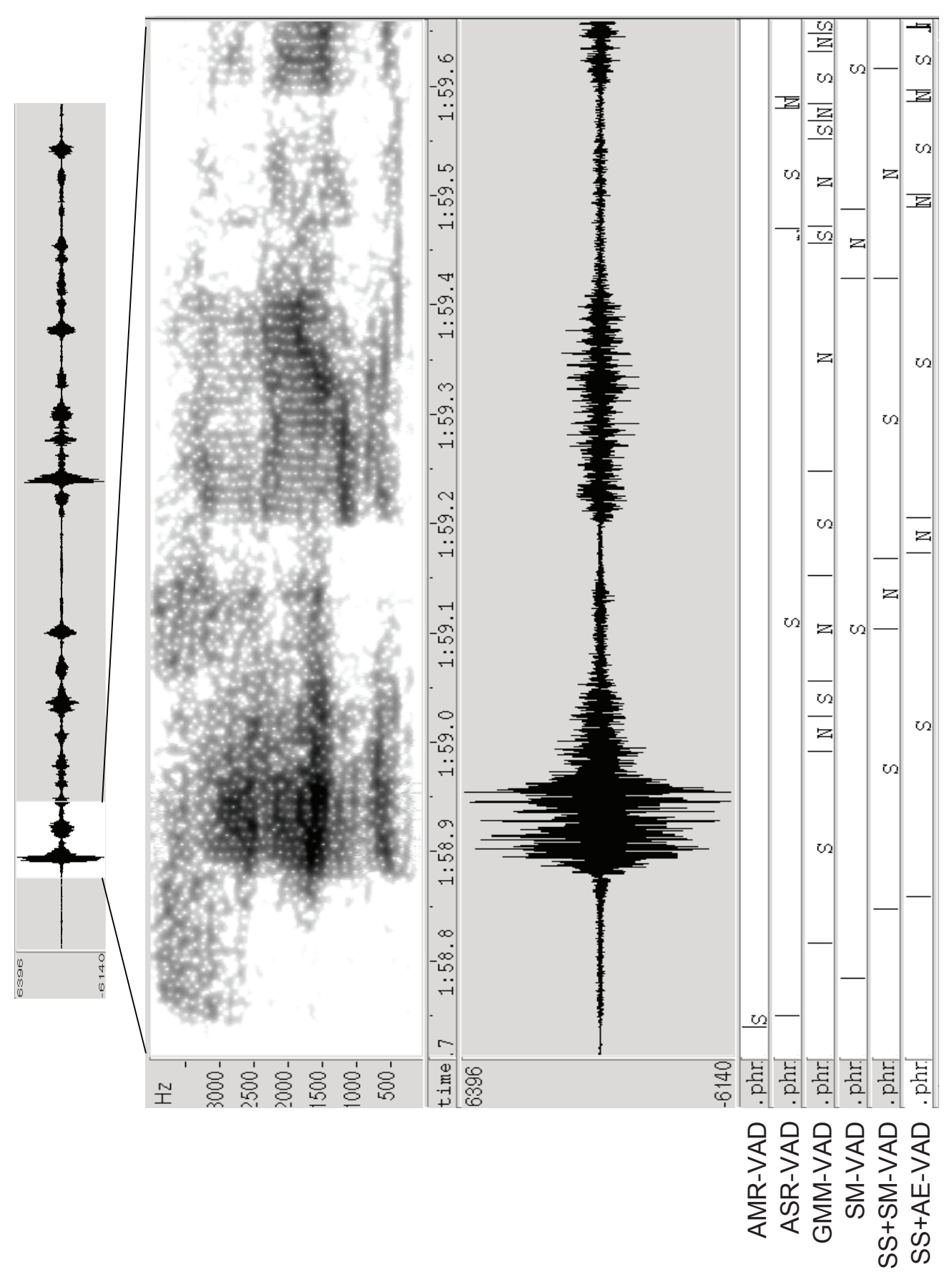

Figure 3: A short segment of low-energy interview speech in NIST 2010 SRE with high-energy spikes. The bottom panel shows the speech (S) and non-speech (N) decisions made by six VADs detailed in 2 ?3rble 1 . 


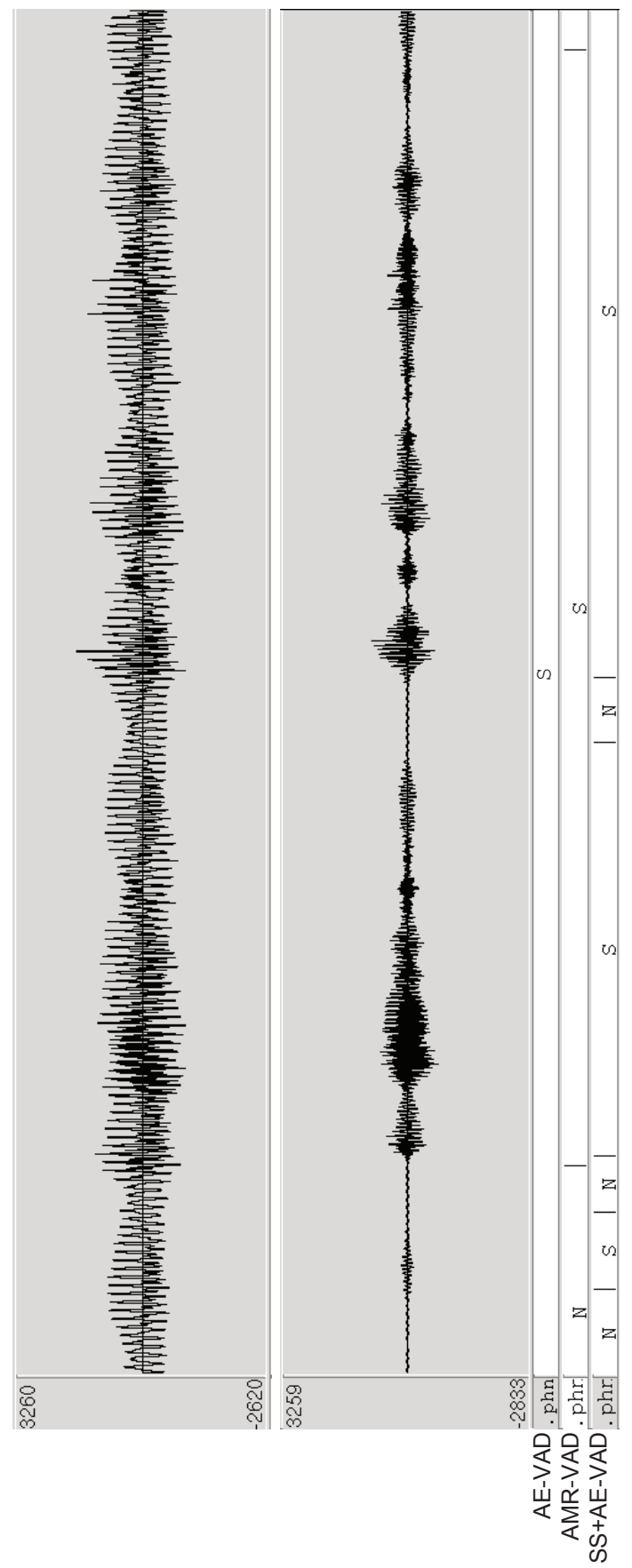

Figure 4: Top: A short segment of low-energy interview speech in NIST 2008 SRE superimposed on periodic background noise. Middle: The same segment after spectral subtraction. Bottom: The VAD decisions (S for speech and $\mathrm{N}$ for nonspeech). See Table 1 for the abbreviationg of the VADs. 


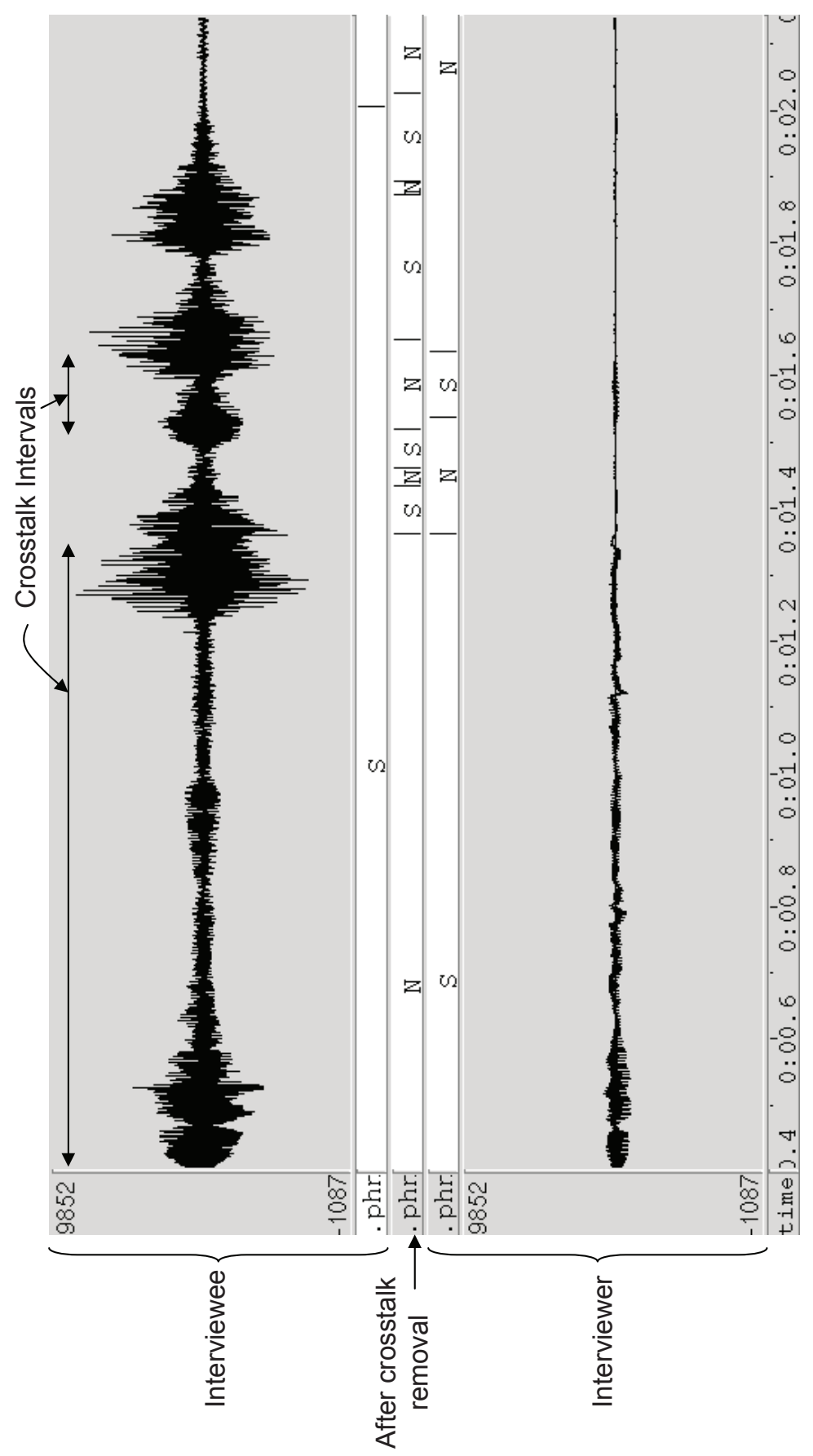

Figure 5: The waveform of a short speech segment from an interviewee and interviewer respectively. The middle panel shows the corresponding VAD results ( $\mathrm{S}$ for speech and $\mathrm{N}$ for non-speech) and the VAD decisions after considering the crosstalk. 


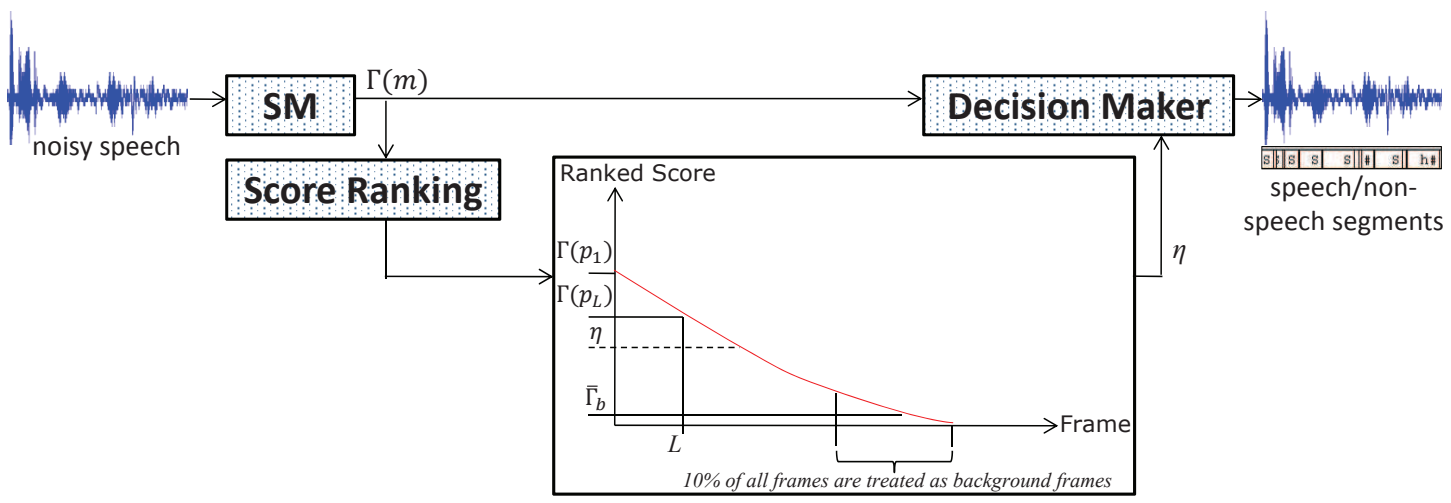

Figure 6: The structure of SM-VAD incorporated with a fixed threshold.

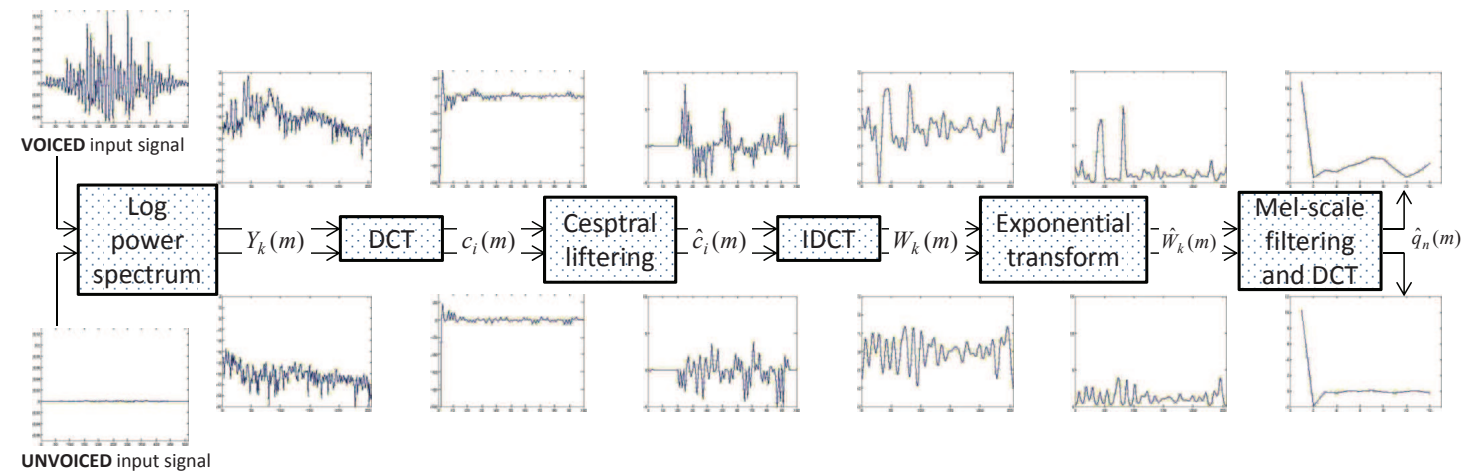

Figure 7: The procedure of extracting the harmonic-structure-based features (after [21]).

[4] Benyassine, A., Shlomot, E., Su, H.Y., 1997. "ITU-T Recommendation G.729 Annex B: A silence compression scheme for use with G.729 optimized for V.70 digital simultaneous voice and data applications". International Telecommunication Union.

[5] Beritelli, F., Casale, S., Ruggeri, G., Serrano, S., 2002. Performance evaluation and comparison of G.729/AMR/Fuzzy voice activity detectors. IEEE Signal Processing Letters 9, 85-88.

[6] Boll, S.F., 1979. Suppression of acoustic noise in speech using spectral subtraction. IEEE Trans. on Acoustics, Speech, and Signal Processing ASSP-27, 113-120.

[7] Campbell, W.M., Sturim, D.E., Reynolds, D.A., 2006a. Support vector 


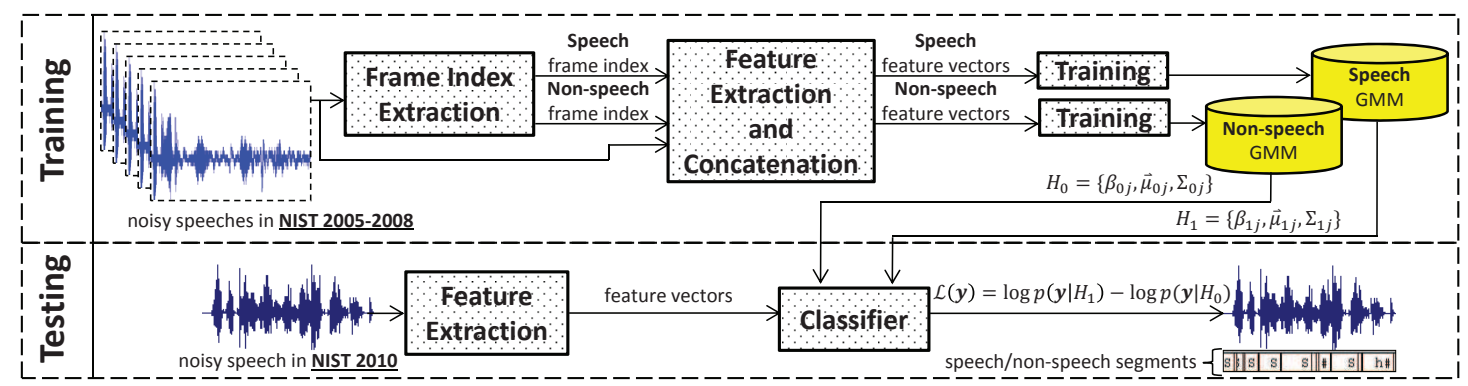

Figure 8: Training and testing of the GMM-based VAD. See Fig. 9 for the algorithm of frame index extraction.

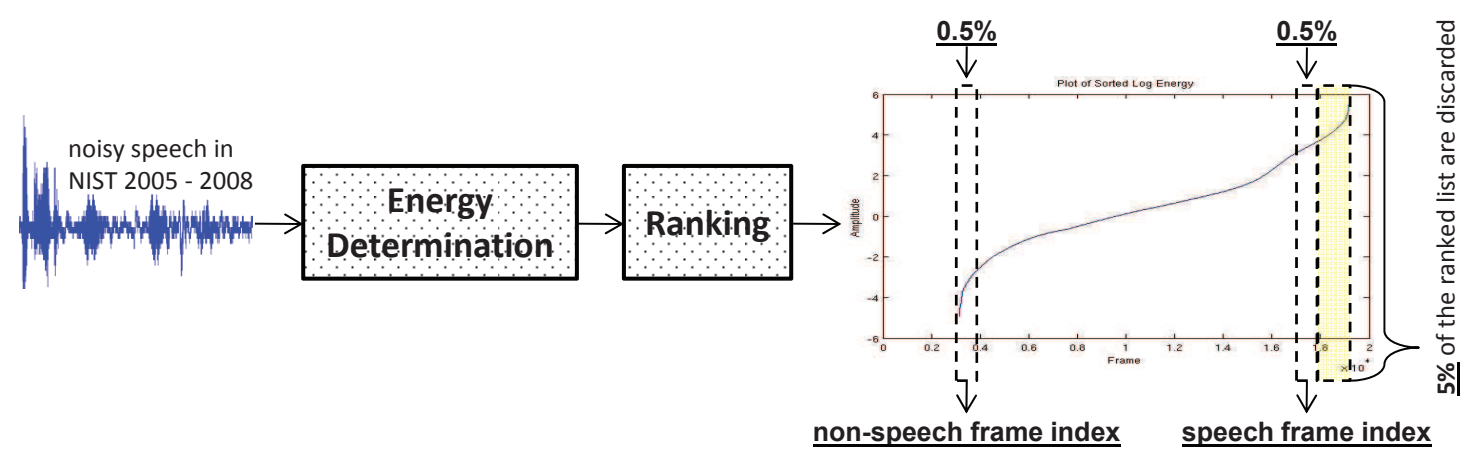

Figure 9: The procedure of extracting the frame indexes representing the speech and non-speech segments in the processing block "Frame Index Extraction" in Fig. 8.

machines using GMM supervectors for speaker verification. IEEE Signal Processing Letters 13, 308-311.

[8] Campbell, W.M., Sturim, D.E., Reynolds, D.A., Solomonoff, A., 2006b. SVM based speaker verification using a GMM supervector kernel and NAP variability compensation, in: Proc. ICASSP, Toulouse, France. pp. $97-100$.

[9] Chang, J.H., Kim, N.S., Mitra, S.K., 2006. Voice activity detection based on multiple statistical models. IEEE Trans. on Signal Processing 54, 1965-1976.

[10] Chengalvarayan, R., 1999. Robust energy normalization using speech/non-speech discriminator for German connected digit recognition, in: Proc. EUROSPEECH 1999, Budapest, Hungary. pp. 61-64. 


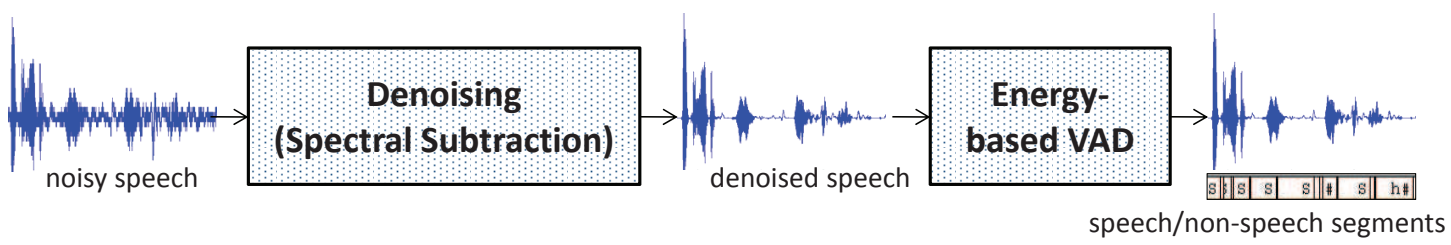

Figure 10: The structure of the proposed VAD for NIST SREs.

[11] Cornu, E., Sheikhzadeh, H., Brennan, R.L., Abutalebi, H.R., Tam, E.C.Y., Iles, P., Wong, K.W., 2003. ETSI AMR-2 VAD: evaluation and ultra low-resource implementation, in: Acoustics, Speech, and Signal Processing (ICASSP'03), Hong Kong. pp. 585-588.

[12] Dalmasso, E., Castaldo, F., Laface, P., Colibro, D., Vair, C., 2009. Loquendo - politecnico di torino's 2008 NIST speaker recognition evaluation system, in: Acoustics, Speech and Signal Processing, 2009. ICASSP 2009, Taipei. pp. 4213-4216.

[13] Davis, S.B., Mermelstein, P., 1980. Comparison of parametric representations for monosyllabic word recognition in continuously spoken sentences. IEEE Trans. on ASSP 28, 357-366.

[14] Dehak, N., Kenny, P., Dehak, R., Dumouchel, P., Ouellet, P., 2011. Front-end factor analysis for speaker verification. IEEE Trans. on $\mathrm{Au}-$ dio, Speech, and Language Processing 19, 788-798.

[15] Deller Jr, J.R., Proakis, J.G., Hansen, J.H.L., 1993. Discrete-time Processing of Speech Signals. Macmillan Pub. Company.

[16] Ephraim, Y., Malah, D., 1984. Speech enhancement using a minimummean square error short-time spectral amplitude estimator. IEEE Transactions on Acoustics, Speech and Signal Processing 32, 1109-1121.

[17] Ephraim, Y., Malah, D., 1985. Speech enhancement using a minimum mean-square error log-spectral amplitude estimator. IEEE Transactions on Acoustics, Speech and Signal Processing 33, 443-445.

[18] ETSI, 1999. Voice activity detector (VAD) for adaptive multi-rate (AMR) speech traffic channels, ETSI EN 301708 v7.1.1.

[19] ETSI, 2001-03. Universal Mobile Telecommunication Systems (UMTS); Mandatory Speech Codec speech processing functions, AMR speech codec; Voice Activity Detector VAD. etsi ts 126094 v4.00 edition. 


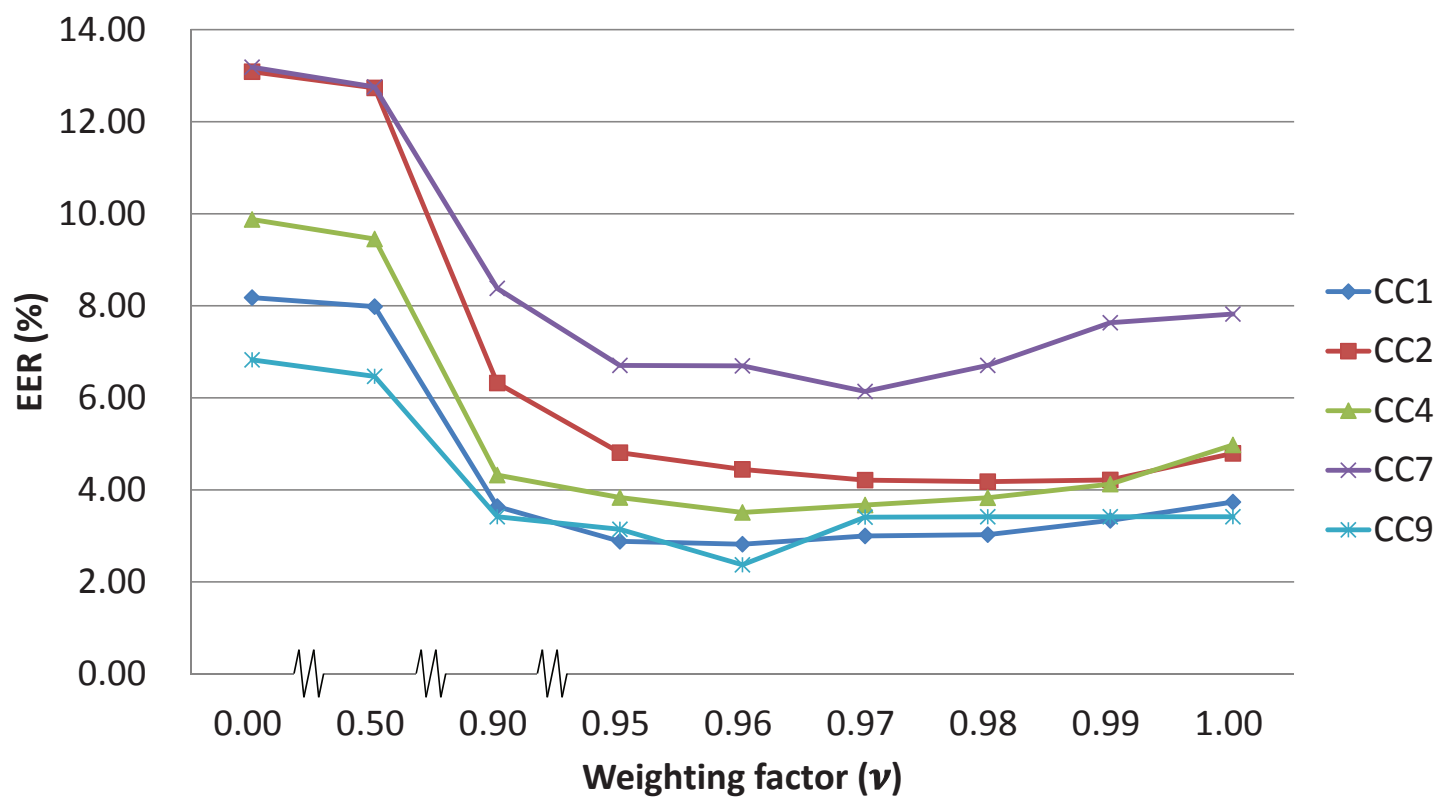

Figure 11: Equal error rate of GMM-SVM against weighting factor $\nu$ in Eq. 9 under Common Conditions (CC) 1, 2, 4, 7 and 9 in NIST'10 (male). SS+AE-VAD (see Table 1) was used in all cases. 


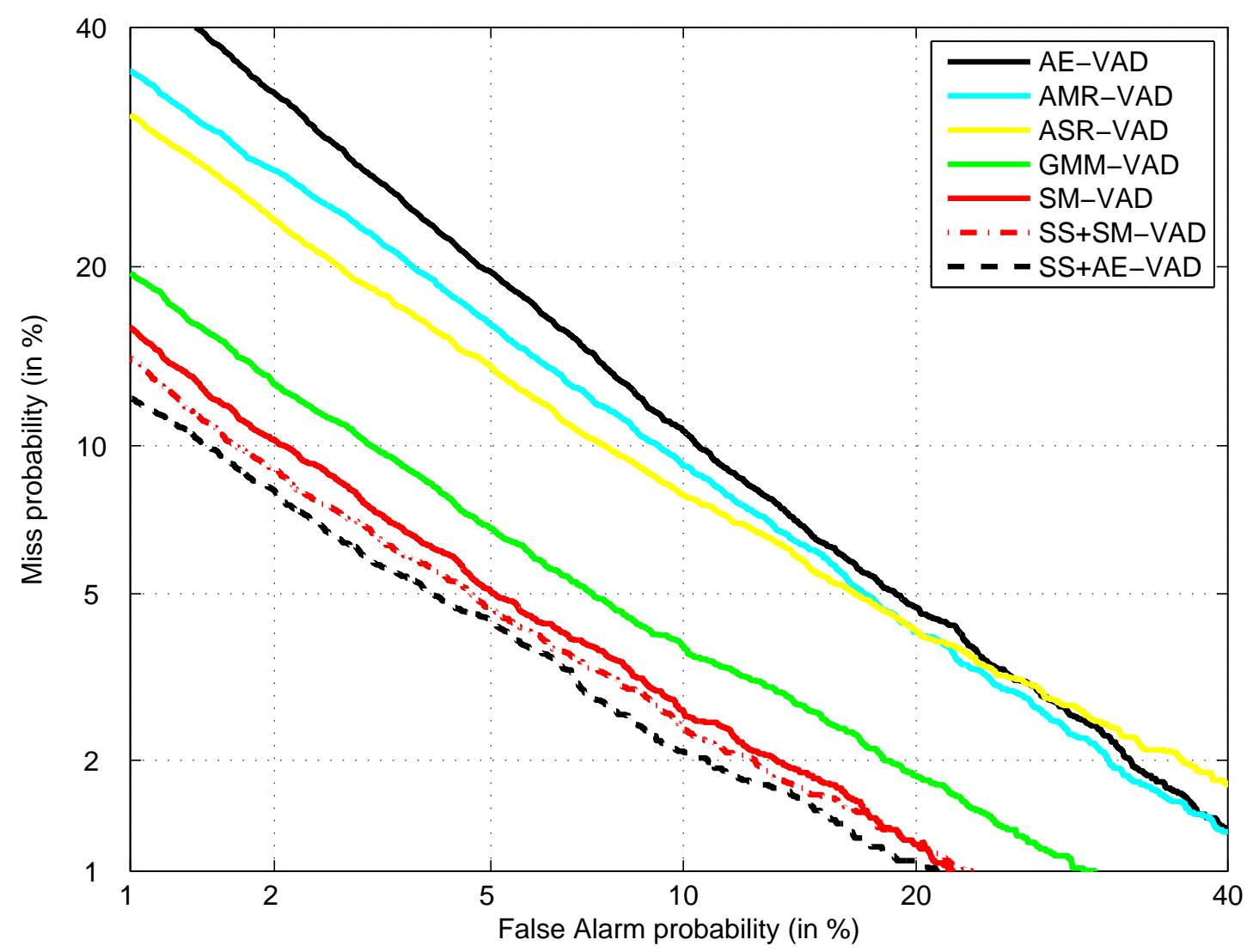

Figure 12: DET performance of GMM-SVM systems for all trials involving interview-style speech in NIST'10 (male). Labels in the legend are arranged in descending EER. Refer to Table 1 for the definitions of different VADs in the legend.

[20] Freeman, D.K., Cosier, G., Southcott, C.B., Boyd, I., 1989. The voice activity detector for the Pan-European digital cellular mobile telephone service, in: Acoustics, Speech, and Signal Processing, 1989. ICASSP-89, Glasgow, UK. pp. 369-372.

[21] Fukuda, T., Ichikawa, O., Nishimura, M., 2010. Long-term spectrotemporal and static harmonic features for voice activity detection. IEEE Journal of Selected Topics in Signal Processing 4, 834-844.

[22] Garcia-Romero, D., Espy-Wilson, C., 2011. Analysis of i-vector length 
normalization in speaker recognition systems, in: Interspeech'2011, pp. 249-252.

[23] Ghosh, P., Tsiartas, A., Narayanan, S., 2011. Robust voice activity detection using long-term signal variability. Audio, Speech, and Language Processing, IEEE Transactions on 19, 600-613.

[24] Góriz, J.M., Ramíez, J., Lang, E.W., Puntonet, C.G., Turias, I., 2010. Improved likelihood ratio test based voice activity detector applied to speech recognition. Speech Communication 52, 664-677.

[25] Gu, L., Rose, K., 2001. Perceptual harmonic cepstral coefficients for speech recognition in noisy environment, in: Acoustics, Speech, and Signal Processing, 2001. Proceedings. (ICASSP '01). 2001 IEEE International Conference on, Salt Lake City, UT, USA. pp. 125-128.

[26] Hautamaki, V., Tuononen, M., Niemi-Laitinen, T., Franti, P., 2007. Improving speaker verification by periodicity based voice activity detection, in: Proc. 12th Int. Conf. Speech and Computer (SPECOM'2007), Moscow. pp. 645-650.

[27] Hirsch, H., Pearce, D., 2000. The Aurora experimental framework for the performance evaluation of speech recognition systems under noisy conditions, in: ASR2000-Automatic Speech Recognition: Challenges for the new Millenium ISCA Tutorial and Research Workshop (ITRW), Paris. pp. 181-188.

[28] Kinnunen, T., Saastamoinen, J., Hautamaki, V., Vinni, M., Franti, P., 2009. Comparing maximum a posteriori vector quantization and Gaussian mixture models in speaker verification, in: Acoustics, Speech and Signal Processing, 2009. ICASSP 2009, Taipei. pp. 4229-4232.

[29] Kitaoka, N., Yamamoto, K., Kusamizu, T., Nakagawa, S., Yamada, T., Tsuge, S., Miyajima, C., Nishiura, T., Nakayama, M., Denda, Y., et al., 2007. Development of VAD evaluation framework CENSREC-1-C and investigation of relationship between VAD and speech recognition performance, in: Automatic Speech Recognition \& Understanding, 2007, ASRU IEEE Workshop on, pp. 607-612.

[30] Li, Q., Zheng, J.S., Tsai, A., Zhou, Q., 2002. Robust endpoint detection and energy normalization for real-time speech and speaker recognition. Speech and Audio Processing, IEEE Transactions on 10, 146 -157. 
[31] Mak, M.W., Yu, H.B., 2010. Robust voice activity detection for interview speech in nist speaker recognition evaluation, in: Proc. APSIPA ASC 2010, Singapore.

[32] Marciniak, T., Rochowniak, R., Dabrowski, A., 2008. Subband wavelet signal denoising for voice activity detection, in: Signal Processing Algorithms, Architectures, Arrangements, and Applications (SPA), 2008, pp. 93-96.

[33] Martin, A., Doddington, G., Kamm, T., Ordowski, M., Przybocki, M., 1997. The DET curve in assessment of detection task performance, in: Proc. Eurospeech'97, pp. 1895-1898.

[34] Martin, A., Greenberg, C. (Eds.), 2010a. NIST SRE10 workshop, NIST Multimodal Information Group, Brno, Czech Republic.

[35] Martin, A.F., Greenberg, C.S., 2010b. The NIST 2010 speaker recognition evaluation, in: Interspeech, Japan. pp. 2726-2729.

[36] Marzinzik, M., Kollmeier, B., 2002. Speech pause detection for noise spectrum estimation by tracking power envelope dynamics. IEEE Transactions on Speech and Audio Processing 10, 109-118.

[37] Nemer, E., Goubran, R., Mahmoud, S., 2001. Robust voice activity detection using higher-order statistics in the LPC residual domain. IEEE Transcations on Speech and Audio Processing 9, 217-231.

[38] Pelecanos, J., Sridharan, S., 2001. Feature warping for robust speaker verification, in: Proc. Odyssey: The Speaker and Language Recognition Workshop, Crete, Greece. pp. 213-218.

[39] Ramirez, J., Gorriz, J.M., Segura, J.C., 2007a. Robust Speech Recognition and Understanding. I-Tech, Vienna, Austria. chapter Voice activity detection. Fundamentals and speech recognition system robustness. pp. $1-22$.

[40] Ramirez, J., Segura, J., Benitez, C., de La Torre, A., Rubio, A., 2004. Voice activity detection with noise reduction and long-term spectral divergence estimation, in: Acoustics, Speech, and Signal Processing, 2004. Proceedings.(ICASSP'04). IEEE International Conference on, pp. 1094-1097. 
[41] Ramirez, J., Segura, J.C., Gorriz, J.M., Garcia, L., 2007b. Improved voice activity detection using contextual multiple hypothesis testing for robust speech recognition. IEEE Trans. Audio Speech Language Processing 15, 2177-2189.

[42] Reynolds, D.A., Quatieri, T.F., Dunn, R.B., 2000. Speaker verification using adapted Gaussian mixture models. Digital Signal Processing 10, $19-41$.

[43] Sangwan, A., Chiranth, M.C., Jamadagni, H.S., Sah, R., Prasad, R.V., Gaurav, V., 2002. VAD techniques for real-time speech transmission on the internet, in: IEEE International Conference on High-Speed Networks and Multimedia Communications, pp. 46-50.

[44] Sohn, J., Kim, N.S., Sung, W., 1999. A statistical model-based voice activity detection. IEEE Signal Processing Letters 6, 1-3.

[45] Sun, H., Ma, B., Li, H., 2008. An Efficient Feature Selection Method for Speaker Recognition, in: ISCSLP'08, pp. 1-4.

[46] Sun, H., Nwe, T., Ma, B., Li, H., 2009. Speaker diarization for meeting room audio, in: Proc. Interspeech, pp. 900-903.

[47] Tanyer, S.G., Ozer, H., 2000. Voice activity detection in nonstationary noise. IEEE Transcations on Speech and Audio Processing 8, 478-482.

[48] Torre, A.D.L., Ramirez, J., Benitez, C., Segura, J.C., Garcia, L., Rubio, A.J., 2006. Noise robust model-based voice activity detection, in: Interspeech, Pittsburgh, Pennsylvania. pp. 1954-1957.

[49] Tucker, R., 1992. Voice activity detection using a periodicity measure. Communications, Speech and Vision, IEE Proceedings I 139, 377-380.

[50] Varela, Ó., San-Segundo, R., Hernández, L., 2011. Combining pulsebased features for rejecting far-field speech in a HMM-based voice activity detector. Computers \& Electrical Engineering 37, 589-600.

[51] Virag, N., 1999. Single channel speech enhancement based on masking properties of the human auditory system. IEEE Trans. on Speech and Audio Processing 7, 126-137.

[52] Vlaj, D., Kacic, Z., Kos, M., 2012. Voice activity detection algorithm using nonlinear spectral weights, hangover and hangbefore criteria. Computers \& Electrical Engineering 38, 1820-1836. 
[53] Woo, K.H., Yang, T.Y., Park, K.J., Lee, C.Y., 2000. Robust voice activity detection algorithm for estimating noise spectrum. Electronics Letters 36, 180-181.

[54] Yu, H., Mak, M., 2011. Comparison of voice activity detectors for interview speech in nist speaker recognition evaluation, in: Interspeech, pp. 2353-2356. 